\title{
THE BUCKLING OF A PRESTRESSED CIRCULAR PLATE*
}

\author{
BY \\ LI-CHIEH CHEN \\ Northeastern University
}

1. Introduction. We consider a thin circular elastic plate of constant thickness. The plate is prestressed in the following manner: a slit is cut in the plate, a wedge of small angle is inserted in such a way as to open out the plate fanwise, and the wedge is then welded to the plate along its edges, as indicated in Fig. 1. If the plate is then subjected to additional compressive stresses in its plane, it will not buckle until these stresses are more than three times as large as they would be for buckling of the initially unstressed plate, if the angle is properly chosen.

In a paper by Stoker [1], the critical values for the angle $\alpha$ at which buckling occurs when the applied pressure is zero were calculated. Afterwards a rough estimate for the critical angle $\alpha_{c}$ at which the lowest critical compressive stress attains a maximum was obtained.

It is the purpose of the present investigation to improve the above results and to calculate the critical applied pressure which corresponds to a given wedge angle $\alpha$. In particular, an investigation of the singularity of the solution of the differential equations is carried out; this then aids in obtaining numerical results.

The mathematical formulation of the problem is based on the equation developed by von Kármán [2]. The deflection of the bent middle surface of the plate was taken in the form

$$
w(r, \theta)=f(r) \cos n \theta, \quad n=0,1,2, \cdots,
$$

in which the integer $n$ refers to the number of diametral nodes. The von Kármán equation is then reduced to a fourth-order ordinary differential equation. This linear homogeneous ordinary differential equation, plus boundary conditions, constitutes an eigenvalue problem, where the eigenvalue corresponds to either the wedge angle $\alpha$ (if the compressive pressure is taken to be zero) or the compressive pressure $p$ (for any fixed angle $\alpha$ ). In both cases the smallest wedge angle $\alpha$ for $p=0$ (or the smallest pressure $p$ for a fixed $\alpha$ ) at which the plane state of the clamped circular plate becomes unstable is determined by solving the ordinary differential equation numerically. For this purpose a CDC 6600 digital computer was used. The method used in obtaining the numerical solution is the so-called "shooting technique"; that is, parameters are introduced as part of the initial conditions. Then these parameters are adjusted so as to make the solutions satisfy the proper boundary conditions. With the help of other techniques the desired results are obtained.

* Received April 2, 1971. 

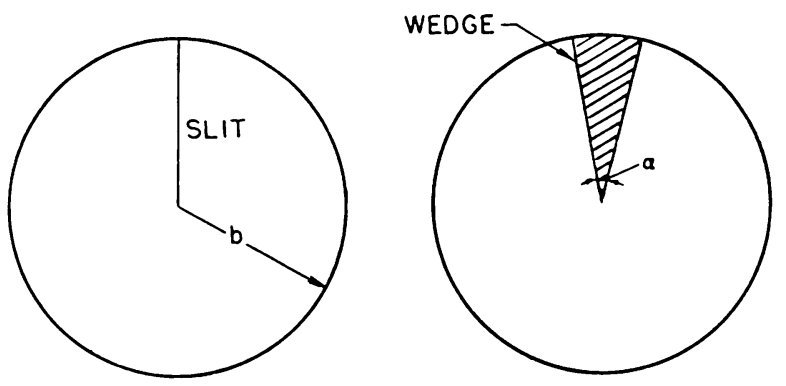

FIG. 1. Circular plate with wedge inserted.

2. Mathematical formulation. The plane stresses ${ }^{1}$ due to the uniform compressive stress $p$ and the initial stresses which arise either from the insertion of a wedge of angle $\alpha$ into a radial slit in the plate or from the removal of such a wedge followed by drawing the edges together and welding them are

$$
\begin{aligned}
& \sigma_{r}=-p-\frac{\alpha E}{4 \pi} \log \left(\frac{r}{b}\right) \\
& \sigma_{\theta}=-p-\frac{\alpha E}{4 \pi}\left[1+\log \frac{r}{b}\right], \tau_{r \theta}=0 .
\end{aligned}
$$

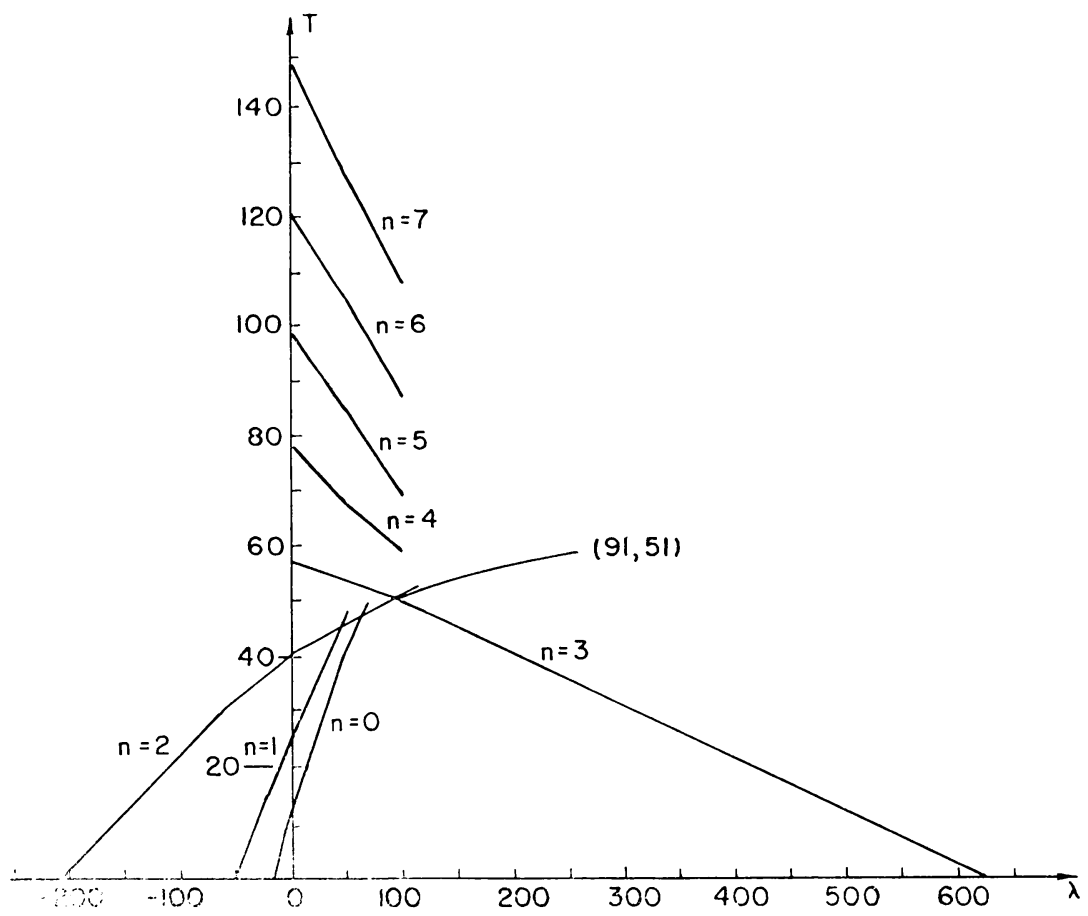

FIG. 2. Curves for critical values of $\lambda$ vs. $T$ for various values of $n$.

1 The initial stresses of the plate are obtained from those of a ring (see [3, pp. 63 and 69]) by letting the inner radius of the ring approach zero. 
( $\alpha>0$ corresponds to the insertion of a wedge; $\alpha<0$ corresponds to a removal of a wedge.)

The deflection $w(r, \theta)$ of the bent middle surface of the plate is taken in the form

$$
w(r, \theta)=f(r) \cos n \theta, \quad n=1,2,3, \cdots .
$$

We substitute $w(r, \theta)$ into von Kármán's equation, and arrive at

$$
\begin{aligned}
\frac{d^{4} f}{d x^{4}}-\frac{2}{x} \frac{d^{3} f}{d x^{3}}-\left[\frac{1+2 n^{2}}{x^{2}}-(T\right. & +\lambda \log x)] \frac{d^{2} f}{d x^{2}} \\
& +\left\{\frac{1+2 n^{2}}{x^{3}}+\frac{1}{x}[T+\lambda(1+\log x)]\right\} \frac{d f}{d x} \\
& +\left\{\frac{n^{4}-4 n^{2}}{x^{4}}-\frac{n^{2}}{x^{2}}[T+\lambda(1+\log x)]\right\} f(x)=0
\end{aligned}
$$

where $x=r / b, x \in[0,1], T=p h b^{2} / N, \lambda=\alpha E h b^{2} / 4 \pi N, N=E h^{3} / 12\left(1-\nu^{2}\right), \nu=$ Poisson's ratios and $h=$ thickness of the plate.

For $n=0$, the differential equation (2) can be transformed into a second-order ordinary differential equation by letting $q(x)=(1 / x)(d f / d x)$; we have

$$
\frac{d^{2} q}{d x^{2}}+\frac{3}{x} \frac{d q}{d x}+(T+\lambda \log x) q=0 .
$$

The boundary condition for a clamped edge at $x=1$ is

$$
q(1)=0 .
$$

The regularity condition at $x=0$ is

$$
\lim _{x \rightarrow 0} x(d q / d x)=0 .
$$

To find the behavior of the solution of Eq. (3) at $x=0$, where there is an irregular singularity, we transform the point $x=0$ to infinity by letting $t=-\log x$. Eq. (3) becomes

$$
\frac{d^{2} q}{d t^{2}}-2 \frac{d q}{d t}-\left(\lambda t e^{-2 t}-T e^{-2 t}\right) q=0 .
$$

The boundary and regularity conditions are

$$
\begin{aligned}
q(0) & =0, \\
\lim _{t \rightarrow \infty}(d q / d t) & =0 .
\end{aligned}
$$

It can be proved [5, p. 127] that there exists a solution $q(t)$ of Eq. (6) such that

$$
q(t) \sim \exp \left[\frac{\lambda}{4}\left(\frac{1}{2}-\frac{1}{2} e^{-2 t}-t e^{-2 t}\right)+\frac{T}{2}\left(1-e^{-2 t}\right)\right]
$$

and $q(t)$ satisfies the condition (8). Therefore, we have the asymptotic behavior of the solution of (3)

$$
q(x) \sim \exp \left[\frac{\lambda}{4}\left(\frac{1}{2}-\frac{1}{2} x^{2}+x^{2} \log x\right)+\frac{T}{2}\left(1-x^{2}\right)\right]
$$


which satisfies Eq. (5).

For the general case $n=1,2,3, \cdots$ as denoted in Eq. (2), the boundary conditions for a clamped edge are

$$
f(1)=0, \quad d f(1) / d x=0 .
$$

The regularity conditions are

$$
\begin{aligned}
f(0) & =0, \\
d f(0) / d x & =0, \quad \text { if } \quad n \geq 2, \\
d^{2} f(0) / d x^{2} & =0, \quad \text { if } \quad n=1 .
\end{aligned}
$$

At the point $x=0$, the differential equation (2) has an irregular singularity. The asymptotic expansion of the solution at the singular point is derived as follows.

We transform the singularity to infinity by letting $t=-\log x$ and then write the equation in the form

$$
d Y / d t=(A+B(t)) Y(t)
$$

where

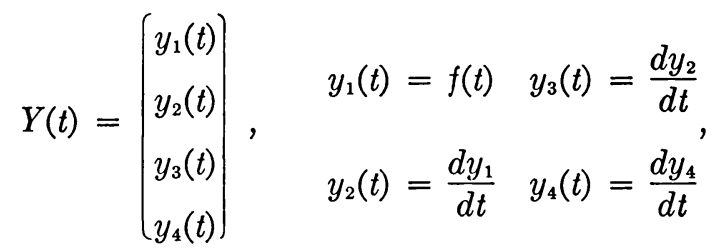

$$
\begin{aligned}
& A=\left(\begin{array}{cccc}
0 & 1 & 0 & 0 \\
0 & 0 & 1 & 0 \\
0 & 0 & 0 & 1 \\
4 n^{2}-n^{4} & 4 n^{2} & 2 n^{2}-4 & -4
\end{array}\right) \\
& B(t)=\left(\begin{array}{cccc}
0 & 0 & 0 & 0 \\
0 & 0 & 0 & 0 \\
0 & 0 & 0 & 0 \\
e^{-2 t} n^{2}[T+\lambda(1-t)] & -e^{-2 t}[T+\lambda(1-t)] & -e^{-2 t}(T-\lambda t) & 0
\end{array}\right) .
\end{aligned}
$$

The characteristic equation of the matrix $A$ is

$$
\beta^{4}+4 \beta^{3}+\left(4-2 n^{2}\right) \beta^{2}-4 h^{2} \beta+\left(n^{4}-4 h^{2}\right)=0 .
$$

The roots of the above equation are

$$
\begin{array}{ll}
h=1, & \beta_{1}=-3, \quad \beta_{2}=-1, \quad \beta_{3}=-1, \quad \beta_{4}=1 \\
n \geq 2, & \beta_{1}^{[n]}=-(n+2), \quad \beta_{2}^{[n]}=-n, \quad \beta_{3}^{[n]}=n-2, \quad \beta_{4}^{[n]}=n .
\end{array}
$$

For $n \geq 2$, all the characteristic roots are simple, and the matrix $B$ has the properties

$$
\lim _{t \rightarrow \infty}\|B\|=0, \quad \lim _{t \rightarrow \infty} \int\|B\| d t<\infty .
$$


It is known [5, p. 45] that the solution of Eq. (12) behaves at infinity as follows:

$$
Y^{[n]}(t) \sim \exp \left(\beta_{k}^{[n]} t\right) \xi_{k}^{[n]}, \quad n=2,3,4, \cdots,
$$

where $\beta_{k}^{[n]}$ are the characteristic roots of the matrix $A$, and $\xi_{k}^{[n]}$ are the corresponding eigenvectors: $\xi_{k}^{[n]}=\left(1, \beta_{k}^{[n]},\left(\beta_{k}^{[n]}\right)^{2},\left(\beta_{k}^{[n]}\right)^{3}\right)$. Thus, for Eq. (2), the corresponding solution for $n=2,3, \cdots$ behaves as follows:

and

$$
f^{[n]}(x) \sim \sum_{k=1}^{4} c_{k}^{[n]} x^{-\beta_{k}[n]}
$$

$$
\frac{d f^{[n]}}{d x} \sim \sum_{k=1}^{4} c_{k}^{[n]}\left(-\beta_{k}^{[n]}\right) x^{\left(-\beta_{k}^{[n]}-1\right)} .
$$

To satisfy the regularity condition (11), $-\beta_{k}^{[n]}-1$ must be 0 , that is, $\beta_{k}^{[n]} \leq 1$.

For the case $n=1$, the $\operatorname{root} \beta=-1$ is not simple; but with the help of a theorem $[6$, p. 92] it can be shown that there exists a solution such that

$$
f^{[1]}(x) \sim c_{1}^{[1]} x^{3}+c_{2}^{[1]} \text { as } x \rightarrow 0 .
$$

Therefore we have the expansion of the solution of Eq. (2):

$$
f^{[n]}(x) \sim c_{1}^{[n]} x^{[n+2]}+c_{2}^{[n]} x^{n}, \quad n=1,2,3, \cdots .
$$

3. Numerical solutions. The method used to solve this eigenvalue problem where the eigenvalue corresponds to either $\lambda$ (for fixed $T$ ) or $T$ (for fixed $\lambda$ ) is known as the "shooting technique". We consider the boundary value problem as an initial value problem with initial condition obtained from the asymptotic expansion.

Cases. $n=1,2,3, \cdots$. For convenience, we transform the singular point $x=0$ to $z=1$, by letting $z=1-x$; then let $y_{1}=f(z), y_{2}=f^{\prime}(z), y_{3}=f^{\prime \prime}(z), y_{4}=f^{\prime \prime \prime}(z)$.

We adjoin to condition (10), which now serves as part of the initial conditions and is in the form $y_{1}(0)=0, y_{2}(0)=0$, the following conditions: $y_{3}(0)=c, y_{4}(0)=1$.

The conditions at $z=1$ are obtained from (9); these are

$$
\begin{aligned}
& y_{1}(1)=0, \\
& y_{2}(1)=0, \quad \text { if } \quad n \geq 2, \\
& y_{3}(1)=0, \quad \text { if } \quad n=1 .
\end{aligned}
$$

For our problem the point $x=1$ is singular. Therefore we integrate at the point $z=1-\epsilon$. From the asymptotic expansion (13) we have

with an error of order $\epsilon$.

$$
\begin{array}{ll}
y_{1}(1-\epsilon)=0, & \\
y_{2}(1-\epsilon)=0, & n \geq 2, \\
y_{3}(1-\epsilon)=0, & n=1,
\end{array}
$$

The parameters are $C$ and $T$ (or $\lambda$ ); they are adjusted so that conditions (14) are satisfied. These conditions are now in the form

$$
\begin{aligned}
& y_{1}(1-\epsilon ; \lambda, T, C)=0, \\
& y_{2}(1-\epsilon ; \lambda, T, C)=0, \quad n \geq 2, \\
& y_{3}(1-\epsilon ; \lambda, T, C)=0, \quad n=1 .
\end{aligned}
$$


In the four-dimensional spaces- $\left(\lambda, T, C, y_{1}\right)$ and $\left(\lambda, T, C, y_{2}\right)$ or $\left(\lambda, T, C, y_{3}\right)$ if $n=1$-we are interested in the space curve in the $(\lambda, T, C)$-space which satisfies $y_{1}=0$ and $y_{2}=0$ (or $y_{3}=0$ if $n=1$ ) simultaneously. For a given $\lambda$, we can obtain the parameters $C$ and $T$. Actually we are only interested in the value $T$, that is, in determining the curve $T(\lambda)$ in the $\lambda T$-plane. The point $T_{0}=\gamma^{2}$, corresponding to $\lambda=0$, i.e. to the case of the unslitted plate (i.e. $\alpha=0$ ), where $\gamma$, in our case, is the smallest zero of $J_{n+1}(x)$. To obtain the curve $T(\lambda)$ we start at a value of $\lambda=\lambda_{1}$ which is near $\lambda_{0}=0$; the corresponding $T\left(\lambda_{1}\right)$ should then be close to $T_{0}$.

Let us fix a value for $\epsilon$ and select four points $\left(T_{11}, C_{11}\right),\left(T_{12}, C_{11}\right),\left(T_{12}, C_{12}\right),\left(T_{11}, C_{12}\right)$ in a $T C$-plane which form the rectangle $R_{1}$. [ $\left.T_{11}, T_{12}\right]$ is chosen such that $T_{0} \in\left[T_{11}, T_{12}\right]$; $\left[C_{11}, C_{12}\right]$ can be chosen arbitrarily as the functions $y_{i}(1-\epsilon ; \lambda, T, C) i=1,2,3$ are linear in $C$ (see Fig. 3).

Using a Runge-Kutta method with a mesh size $m$, we arrive at value of $y_{i}(1-\epsilon$; $\lambda, T, C) i=1,2$ (or 3 if $n=1$ ) at the four given points. In the $y_{1} C$-plane, we look for the intersection of the $C$-axis with the straight line defined by the two points $\left(y_{1}(1-\epsilon\right.$; $\left.\left.\lambda_{1}, T_{11}, C_{11}\right), C_{11}\right)$ and $\left(y_{1}\left(1-\epsilon ; \lambda_{1}, T_{11}, C_{12}\right), C_{12}\right)$. We have thus found a $C=C_{1}^{1}$ at which $y_{1}=0$. Similarly, we look for the intersection of the $C$-axis with the straight line defined by the two points $\left(y_{1}\left(1-\epsilon ; \lambda_{1}, T_{12}, C_{11}\right), C_{11}\right)$ and $\left(y_{1}\left(1-\epsilon ; \lambda_{1}, T_{12}, C_{12}\right), C_{12}\right)$. We have thus found a $C=C_{2}^{1}$ at which $y_{1}=0$. If we apply this procedure to the $y_{2} C$ plane, we can derive $C_{1}^{2}$ and $C_{2}^{2}$ at which $y_{2}=0$. The points $\left(T_{11}, C_{1}^{1}\right),\left(T_{12}, C_{2}^{1}\right)$ and $\left(T_{11}, C_{1}^{2}\right),\left(T_{12}, C_{2}^{2}\right)$ define two straight lines respectively in the $T C$-plane. The intersection of these lines, denoted by $I_{1}$, satisfies the conditions $y_{1}=0$ and $y_{2}=0$; and $I_{1} \in R_{1}$ (if not, we can enlarge the size of $R_{1}$ ) (see Fig. 4).

To refine our estimate of $I_{1}$, we form a new rectangle $R_{2}$ with $I_{1}$ as center and $R_{2} \subset R_{1}$. Following the same procedure, we find an intersection $I_{2}$ such that $I_{2} \in R_{2}$. In this way we derive a sequence of intersections $\left\{I_{k}\right\}$ and a sequence of rectangular regions $\left\{R_{k}\right\}$

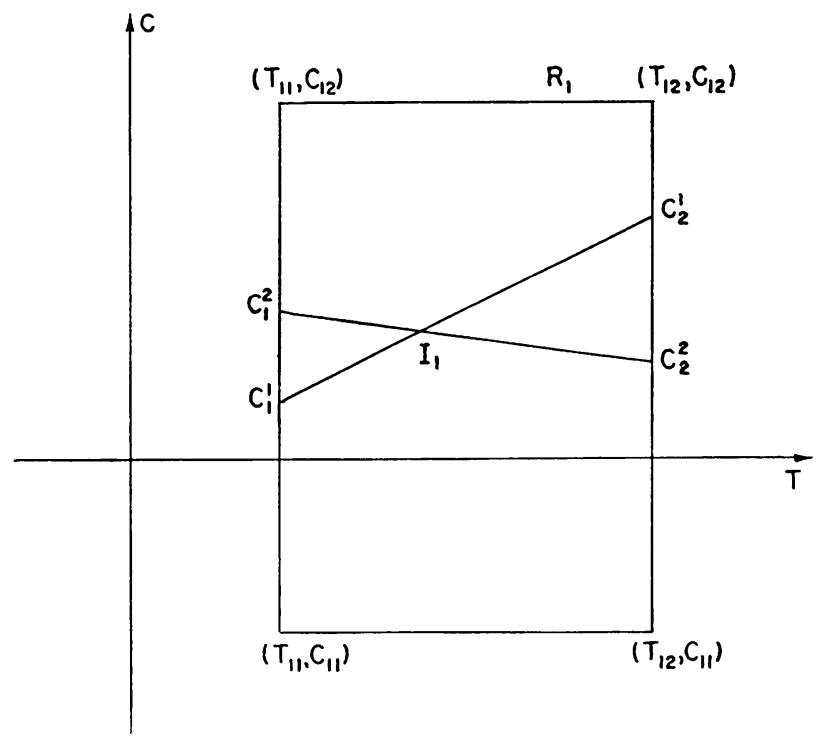

Fig. 3. $T C$-plane at $\lambda=\lambda_{1}$. 


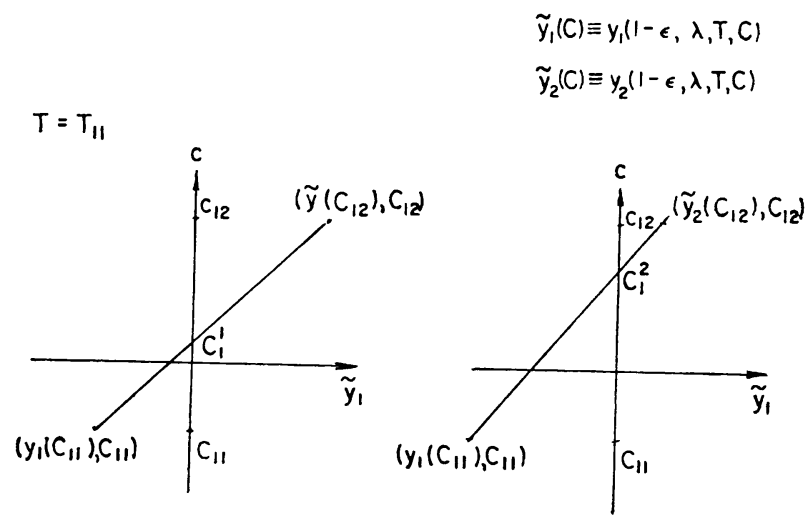

$T=T_{12}$

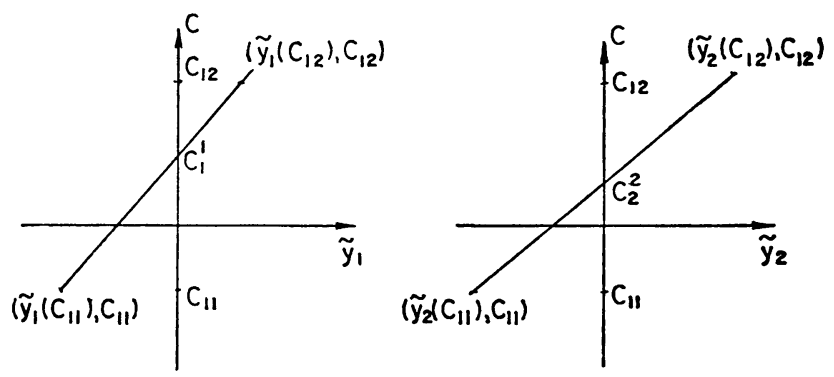

FIG. 4. At $\lambda=\lambda_{1}, \tilde{Y}_{i} C$-planes for fixed $T$.

such that $R_{k} \subset R_{k-1}$ and $I_{k} \in R_{k}$. If $\left\{I_{k}\right\}$ converges to a point $I$ as we let $\epsilon \rightarrow 0$ and the relations hold when $m \rightarrow 0$, then the point $I(T, C)$ is the desired eigenvalue of our problem.

As an example, we consider the case $n=7$ and $\lambda_{1}=100$. Then the point $T_{0}=$ $T(0)=147.5$. Using $T_{0}$ as reference, we let $R_{1}$ be defined by the four points $\left(T_{11}, C_{11}\right)$, $\left(T_{12}, C_{11}\right),\left(T_{12}, C_{12}\right),\left(T_{11}, C_{12}\right)$ where $T_{11}=1, T_{12}=200, C_{11}=-1, C_{12}=1$. A Runge-Kutta method is applied. (In the interval $[0, m]$ we use the mesh $m$; in $[m, 1-\epsilon]$ we use the mesh size $\epsilon$.) The values of $y_{i}(1-\epsilon ; \lambda, T, C)$ are obtained at the four points. And the value of $C$ at which $y_{i}(1-\epsilon ; 100,1, C), i=1,2$, equals zero at $T_{11}=1$ is $C_{1}^{1}=-0.0954, C_{1}^{2}=-0.0953$. The value of $C$ at which $y_{i}(1-\epsilon ; 100,200, C), i=1,2$, equals zero at $T_{12}=200$ is $C_{2}^{1}=-0.1335, C_{2}^{2}=-0.1336$. The two straight lines defined by the two points $\left(T_{11}, C_{1}^{1}\right),\left(T_{12}, C_{2}^{1}\right)$ and $\left(T_{11}, C_{1}^{2}\right),\left(T_{12}, C_{2}^{2}\right)$ respectively have the intersection $I_{1}$ (in the $T C$-plane) $I_{1}=(130.7564,-0.1203)$. We let $R_{2}$ be defined by the points $\left(T_{21}, C_{21}\right),\left(T_{22}, C_{21}\right),\left(T_{22}, C_{22}\right)$ and $\left(T_{21}, C_{22}\right)$, where $T_{21}=110, T_{22}=150$, $C_{21}=-1, C_{22}=1$. The same procedure gives $I_{2}=(111.6393,-0.1190)$. For $R_{3}$, we use $T_{31}=90, T_{32}=120, C_{31}=-1, C_{32}=1$, and $I_{3}=(105.0932,-0.1177)$. We change $m$ to $m=2^{-11}$ but keep $\epsilon=2^{-20}$. Choose $R_{4}$ as $T_{41}=90, T_{42}=110, C_{41}=-0.095$, $C_{42}=-0.121$. Then $I_{4}=(108.1540,-0.1183)$. 
We list further results as follows:

$$
\begin{aligned}
& R_{5} T_{51}=100 \quad C_{51}=-0.119 \quad I_{5}=(108.413,-.1184) \\
& T_{52}=110 \quad C_{52}=-0.114 \\
& R_{6} T_{61}=108 \quad C_{61}=-0.1186 \quad I_{6}=(108.20,-.1184) \\
& T_{62}=109 \quad C_{62}=-0.1183
\end{aligned}
$$

Keep $R_{7}=R_{6}$ and let $m=2^{-13}=1.227 \times 10^{-4}, \epsilon=2^{-20}$. Then $I_{7}=(108.286,-0.1184)$.

Next, we reduce the size of the rectangular region by letting $R_{8}$ be defined by $T_{81}=$ $108.2, T_{82}=108.4, C_{81}=-0.118397, C_{82}=-0.118356$, with $m=2^{-11}, \epsilon=2^{-20}$. Then $I_{8}=(108.3,-0.1184)$. Let $R_{9}=R_{8}$. Change both $m$ and $\epsilon$ to

$$
\begin{array}{lll}
m=2^{-14}, & \epsilon=2^{-21}, & I_{9}=(108.2,-0.1184), \\
m=2^{-15}, & \epsilon=2^{-22}, & I_{10}=(108.2,-0.1184) .
\end{array}
$$

From the above results we obtain the estimate of the eigenvalue $T$. For our purpose it is then reasonable to take $T \in[108,109]$ as a good approximation. For each $n$, we use the same method and obtain the points $(\lambda, T)$ in the $\lambda T$-plane. The points are indicated by a cross sign in Fig. 2.

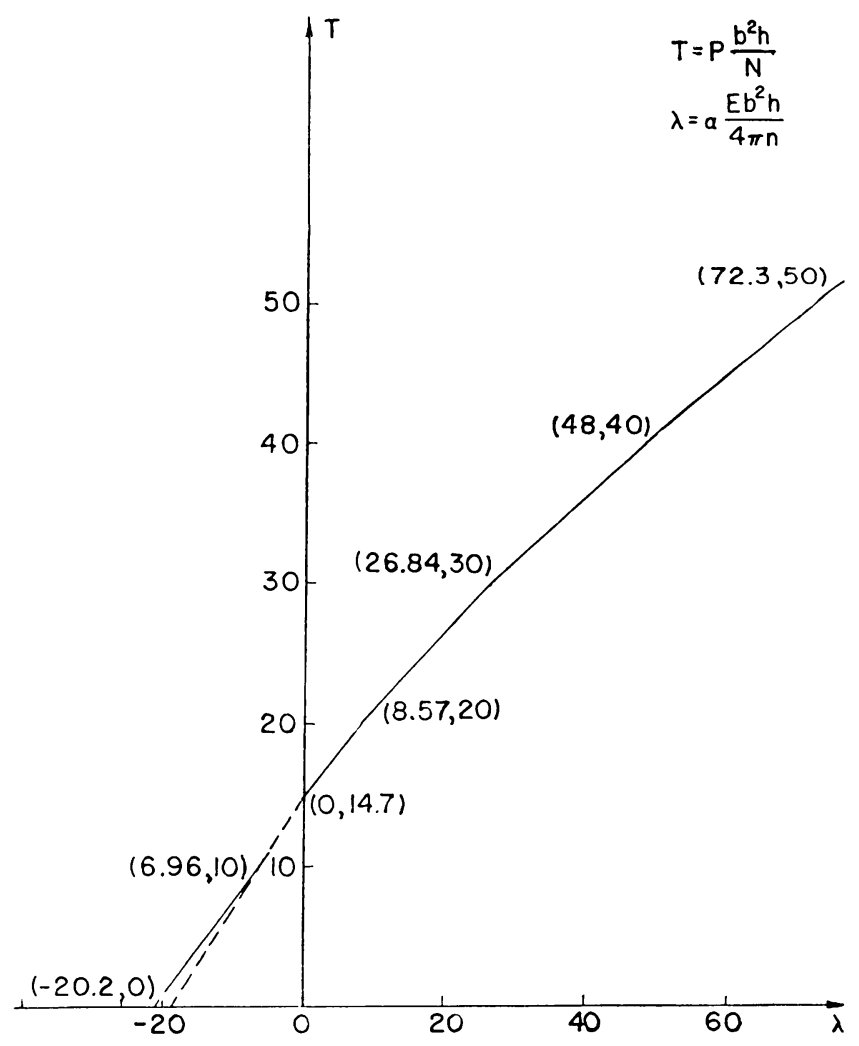

FIg. 5. Curve for critical values of $\lambda$ vs. $T$ for $n=0$. 


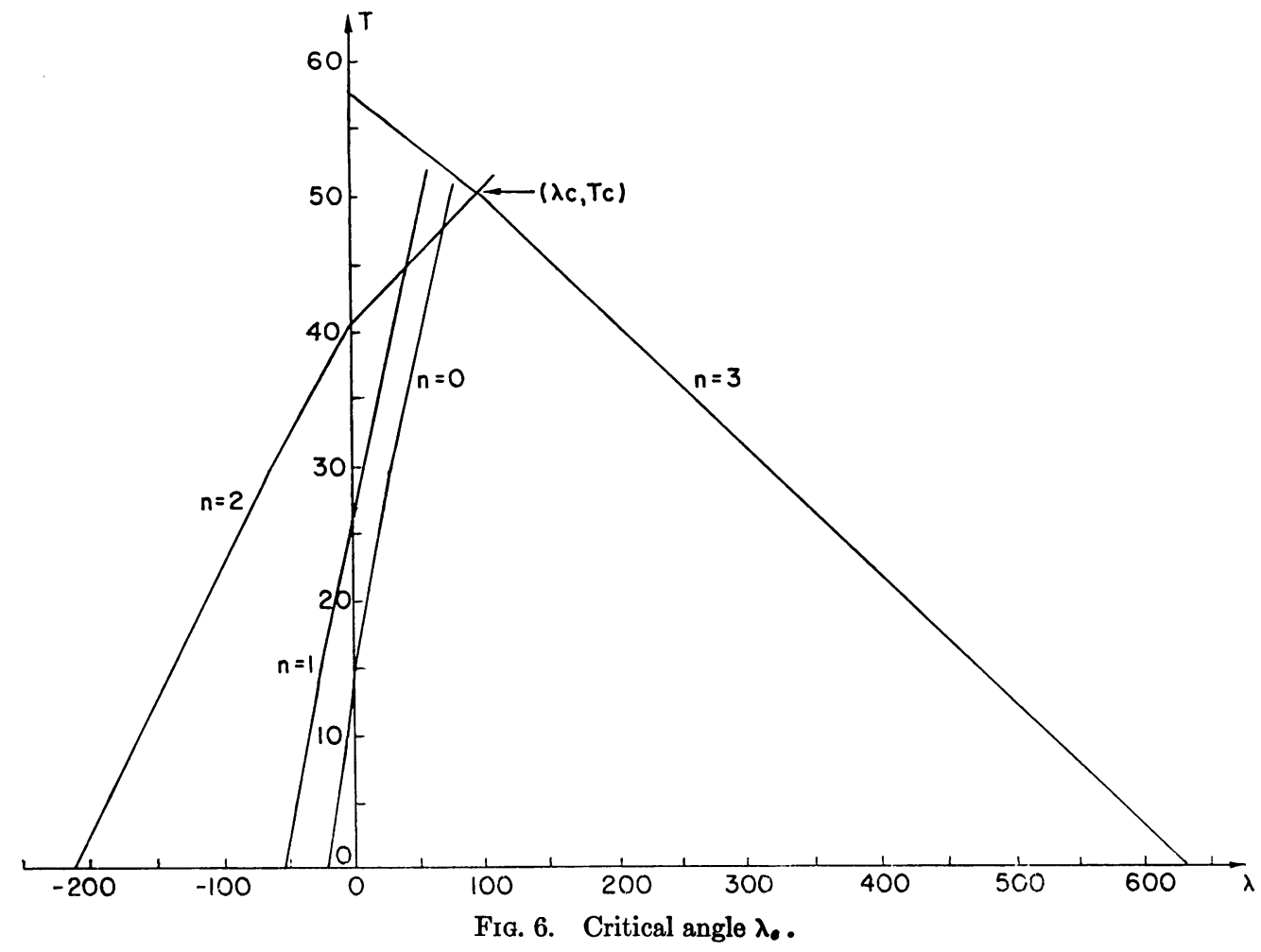

Case. $\quad n=0$. In the three-dimensional space $(\lambda, T, q)$ we are interested in finding the curve $\lambda(T)$ (or $T(\lambda)$ ) in the $\lambda T$-plane which corresponds to $q(1)=0$. Using the same procedure as in cases $n=1,2,3, \cdots$ we list the following results:

$$
\begin{aligned}
& (T, \lambda)=(0,-20.2), \\
& (T, \lambda)=(20,8.6), \\
& (T, \lambda)=(30,26.5), \\
& (T, \lambda)=(40,48.1), \\
& (T, \lambda)=(50,72.3) ;
\end{aligned}
$$

and the curve $\lambda(T)$ is drawn by connecting these points (see Fig. 5).

4. Conclusion. From Fig. 6, we find that by inserting a wedge into the plate (corresponding to positive $\lambda$ ), it is possible to increase the lowest buckling pressure, corresponding to $T=14.7$, to a value which can be estimated as $T=51$ at which the wedge angle $\alpha$ corresponds to $\lambda \cong 90$; thus the critical value of $T$ is more than tripled by inserting a wedge. The critical angle $\alpha_{c}$ would appear to correspond to a value in the neighborhood of $\lambda \cong 90$ and to a buckling mode with three diametral nodes. The wedge angle $\alpha$ is given by the formula

$$
\alpha=\lambda\left(4 \pi N / E h b^{2}\right) .
$$

As $\lambda \cong 90$ and $\nu=0.3$, we have $\alpha \cong 108(h / b)^{2}$. For $n \geq 4$ the calculations make it seem certain that they will not influence the critical value for $\alpha$. 


\section{REFERENCES}

[1] J. J. Stoker, Pre-stressing a plane circular plate to stiffen it against buckling, in Reissner anniversary volume: contributions to applied mechanics, J. W. Edwards, Ann Arbor, Mich., 1948, pp. 268-276

[2] J. J. Stoker, Bending and buckling of elastic plate (lecture)

[3] S. P. Timoshenko and J. N. Goodier, Theory of elasticity, 2nd ed., McGraw-Hill, New York, 1951

[4] J. J. Stoker, Nonlinear elasticity, Gordon and Breach, New York, 1968

[5] R. E. Bellman, Stability theory of differential equations, McGraw-Hill, New York, 1953

[6] W. A. Coppel. Stability and asymptotic behavior of differential equations, Heath, Boston, Mass., 1965 